\title{
Anti-N-methyl D-aspartate receptor encephalitis in childhood
}

\author{
Felippe Borlot, ${ }^{1}$ Mara Lucia F. Santos, ${ }^{2}$ Marcia Bandeira, ${ }^{3}$ Paulo B. Liberalesso, ${ }^{4}$ \\ Fernando Kok, ${ }^{5}$ Alfredo Löhr Jr., ${ }^{6}$ Umbertina C. Reed ${ }^{7}$
}

\begin{abstract}
Objective: To discuss the differential diagnosis of encephalitis beyond that of infectious etiology and to inform pediatricians about the possibility of anti-N-methyl-D-aspartate receptor (NMDAr) encephalitis in children by highlighting its most important clinical features.

Description: Three patients presented with an initial neuropsychiatric syndrome followed by encephalopathy and movement disorder. The initial neuropsychiatric features which developed over days to weeks included a change in personality, anxiety, confusion, and speech regression. This was followed by a choreoathetoid or dystonic movement disorder affecting the orofacial region and the limbs. After the exclusion of the major causes of encephalitis, NMDAr antibodies were identified in serum and cerebrospinal fluid, and neoplasm screening did not detect any tumor. Patients were submitted to immunosuppression, and two of them had a full neurological recovery. One of them still presents a mild dystonic posture in a limb.

Comments: Clinical signs of anti-NMDAr encephalitis in children are similar to those previously described in adults. Tumors are not usually detected by this age. The diagnosis of anti-NMDAr encephalitis must be addressed only after the exclusion of infectious and other recognizable causes of encephalitis. Pediatricians should be aware of this treatable autoimmune condition.
\end{abstract}

J Pediatr (Rio J). 2012;88(3):275-8. Encephalitis, anti-N-methyl-D-aspartate receptor, childhood.

\section{Introduction}

Anti-N-methyl-D-aspartate receptor (NMDAr) encephalitis is a neuropsychiatric syndrome regarded as being caused by immune-mediated processes, once it presents autoantibodies in the serum or in the cerebrospinal fluid (CSF) directed against an epitope located in the extracellular domain of NMDAr. This disorder is often described in the adult population and may be associated with paraneoplastic ovarian teratoma.1,2
NMDAr participates in synaptic transmission and neuronal plasticity. Up- or down-regulation in its activity may explain some of the neurological symptoms present in anti-NMDAr encephalitis, such as seizures, aphasia, and dystonia. Before the identification of the antibodies underlying the pathogenic processes of this entity, patients were often diagnosed as presenting encephalitis of unknown origin if no other known infectious or autoimmune etiology was clarified.

1. Physician. Pediatric neurologist, Hospital Santa Marcelina, São Paulo, Brazil. DFV Neuro team, São Paulo, SP, Brazil.

2. Physician. Pediatric neurologist, Hospital Pequeno Príncipe, Curitiba, PR, Brazil.

3. Physician. Pediatric rheumatologist. MSc in Pediatrics. Investigator and technical responsible, Hospital Pequeno Príncipe, Curitiba, Brazil. Professor of Pediatrics, Universidade Positivo, Curitiba, PR, Brazil.

4. Physician. PhD. Supervisor, Programa de Residência Médica em Neurologia Infantil, Departamento de Neurologia e do Serviço de Neurofisiologia, Hospital Pequeno Príncipe, Curitiba, PR, Brazil.

5. PhD. Tenured professor of Neurology, Faculdade de Medicina, Universidade de São Paulo (USP). Pediatric neurologist, Hospital das Clínicas, Faculdade de Medicina, USP, São Paulo, SP, Brazil.

6. MSc. Chief pediatric neurologist, Setor de Neurologia Infantil, Hospital Pequeno Príncipe, Curitiba, PR, Brazil.

7. PhD. Full professor, Departamento de Neurologia, Faculdade de Medicina, USP, São Paulo, SP, Brazil.

No conflicts of interest declared concerning the publication of this article.

Suggested citation: Borlot F, Santos ML, Bandeira M, Liberalesso PB, Kok F, Löhr Jr. A, et al. Anti-N-methyl D-aspartate receptor encephalitis in childhood. J Pediatr (Rio J). 2012;88(3):275-8.

Manuscript submitted Aug 19 2011, accepted for publication Nov 162011.

http://dx.doi.org/10.2223/JPED.2172 
The frequency of anti-NMDAr encephalitis is not certainly established, but it is assumed that this condition is responsible for almost $1 \%$ of the admissions of young adults in intensive care units. ${ }^{3}$ The largest pediatric series addressing this issue reported 32 patients under the age of 18 years (median: 14 years). ${ }^{4}$ Interestingly, there is no frequent association with paraneoplastic neoplasms at such young ages. ${ }^{4,5}$

The first reports of adult patients presenting anti-NMDAr encephalitis demonstrated the symptomatic evolution, with the onset of changes in mood, behavior and speech followed by seizures, consciousness impairment, dyskinesia, and autonomic instability. In childhood, all of these symptoms were also described, but their presentation may vary: movement disorders were present in $84 \%$ and seizures in $77 \%$ of patients respectively, and prodromal symptoms were non-specific and identified in less than $50 \%$ of a series of 32 young patients. ${ }^{4}$ Indeed, the main differences between presentation in children and adults are the heterogeneity of neurological findings and the association with tumors, which is uncommon in children.

The aim of this case series is to discuss the differential diagnosis of encephalitis beyond that of infectious etiology and to inform pediatricians about the possibility of antiNMDAr encephalitis by highlighting its most important clinical features.

\section{Description of cases}

Informed written consent forms were obtained from all children's parents, and the study was approved by the Research Ethics Committee. Clinical findings of patients are summarized in Table 1.

\section{Patient 1}

A 10-year-old girl presenting a history of acute headache and fever was admitted in intensive care unit with psychomotor agitation and generalized seizure. The seizures became intermittent, but refractory to antiepileptic drugs such as phenytoin, phenobarbital, benzodiazepines, valproic acid, oxcarbazepine, and topiramate. After a week, repetitive involuntary movements emerged: limb chorea and orofacial dyskinesia. The patient developed mood lability, global aphasia, left hemiparesis, and tachycardia alternating with bradycardia. As there was a clinical suspicion of encephalitis, lumbar puncture was performed, revealing normal CSF, after a normal brain computed tomography scan. The electroencephalogram (EEG) showed slow background activity associated with sharp waves in the frontal lobe, which confirmed an involvement of the brain parenchyma and ruled out status epilepticus. Complementary investigation for systemic lupus erythematosus, rheumatic fever, and systemic vasculitis were performed, and the results were negative. Serological evaluation was normal and brain magnetic resonance imaging (MRI) were normal. The patient was empirically treated with intravenous (IV) acyclovir during 21 days, even with consecutively negative polymerase chain reaction studies for viruses in CSF. Seizures were controlled, but other symptoms remained uncontrolled. Finally, antibodies against NMDAr were detected in both CSF and blood by enzyme-linked immunosorbent assay (ELISA). Thereafter, a cycle of IV methylprednisolone (30 $\mathrm{mg} / \mathrm{kg} /$ day) for 5 days was prescribed. As the patient did not respond satisfactorily, IV gammaglobulin $(400 \mathrm{mg} /$ $\mathrm{kg} /$ day) and immunosuppression with cyclophosphamide $\left(800 \mathrm{mg} / \mathrm{m}^{2}\right.$ ) were initiated. Because residual symptoms still remained after 2 months from the therapy with immunoglobulin and cyclophosphamide, two doses of IV rituximab $\left(375 \mathrm{mg} / \mathrm{m}^{2}\right.$ ) with a 14-day interval and prednisone ( $2 \mathrm{mg} / \mathrm{kg} / \mathrm{day}$ ) for 6 months were added. In this meanwhile, no tumor was detected. Nowadays, after 10 months from the onset of the first symptoms, the patient has had a normal intellectual performance and no motor impairment.

\section{Patient 2}

A previously healthy 2-year-old boy developed partial seizures with motor involvement in the absence of fever followed by left hemiparesis. Therapeutic approach using oxcarbazepine and divalproate resolved his seizures. After 2 weeks, though, the patient was unable to speak and uncontrolled movements in the upper limbs emerged. The neurologic examination showed language impairment, preserved comprehension, communication restricted to gestures, dystonic posture in the left hand, and bilateral choreoathetoid movements in the upper limbs. No signs of autonomic dysfunction were detected. Blood tests for autoimmune disorders, thyroid function, and antibodies were normal, as well as CSF and MRI. EEG disclosed a slow background activity with epileptiform discharges in right temporal and median topographies. The patient had no clinical signs or laboratory findings suggesting rheumatologic or psychiatric disorders. No viral agent was identified in CSF. After the exclusion of the main childhood encephalitis agents, NMDAr serum antibodies were identified by ELISA. Neoplasm screening with total abdomen ultrasound, measurement of urinary catecholamine metabolites, and chest computed tomography did not detect any tumor. The patient had a substantial improvement after two cycles of IV methylprednisolone $(30 \mathrm{mg} / \mathrm{kg} /$ day) for 5 days with a 2-month interval, followed by oral prednisone $(1 \mathrm{mg} / \mathrm{kg} /$ day). Three months after the onset of symptoms, language and communication abilities were normal. The patient presented a full recovery of chorea, but a mild dystonic posture in the left hand persisted. 
Table 1 - Patients' characteristics

\begin{tabular}{|c|c|c|c|}
\hline & Patient 1 & Patient 2 & Patient 3 \\
\hline Age of onset of symptoms/gender & 10 years, female & 2 years, male & 3 years, female \\
\hline Prodrome & Fever and headache & No & Severe abdominal pain \\
\hline Consciousness impairment & Yes & Yes & Yes \\
\hline Seizures & $\begin{array}{l}\text { Tonic-clonic seizure } \\
\text { (EEG: frontal discharges) }\end{array}$ & $\begin{array}{l}\text { Complex partial motor seizure } \\
\text { (EEG: temporal discharges) }\end{array}$ & No seizures \\
\hline Speech disturbances & Aphasia & Aphasia & Aphasia \\
\hline Movement disorders & Chorea, orofacial dyskinesia & Dystonia, choreathetosis & Orofacial dyskinesia \\
\hline Cerebrospinal fluid & Normal & Normal & $\begin{array}{l}\text { Lymphocytic pleocytosis } \\
\left(11 \text { cells } / \mathrm{mm}^{3}\right) \text {, normal } \\
\text { protein and glucose } \\
\text { concentrations }\end{array}$ \\
\hline Brain MRI & Normal & Normal & Normal \\
\hline Tumor & Not found & Not found & Not found \\
\hline Treatment & $\begin{array}{l}\text { Steroids }+ \text { immunoglobulin }+ \\
\text { cyclophosphamide }+ \text { rituximab }\end{array}$ & Steroids & $\begin{array}{l}\text { Steroids + immunoglobulin } \\
+ \text { cyclophosphamide }\end{array}$ \\
\hline Outcome & Full neurological recovery* & Substantial improvement* & Full neurological recovery* \\
\hline
\end{tabular}

$E E G=$ electroencephalogram; $M R I=$ magnetic resonance imaging.

* According to Florance et al., ${ }^{4}$ patients were considered to have "full neurological recovery" if they were able to return to all their activities; "substantial improvement" if they returned to their homes with mild deficits and were improving; and "limited improvement" if they were at home, in the hospital, or in a rehabilitation center with minimal change in the neurological status 3 months after neurological symptom presentation.

\section{Patient 3}

A 3-year-old girl with unremarkable previous medical history presented severe abdominal pain followed by mood oscillation, inconsolable crying, agitation, and insomnia alternating with drowsiness 3 days before being admitted into the hospital. The patient did not present seizure, motor deficits or meningism. She underwent a spinal tap and the CSF showed the following results: 11 cells/ $\mathrm{mm}^{3}$ and normal protein and glucose levels. Fungal and bacterial cultures were negative. Brain MRI was normal. EEG showed slow background activity without epileptiform discharges. After the diagnosis of encephalitis, the patient was empirically treated with acyclovir for 21 days. Polymerase chain reaction for herpes simplex was negative. The patient developed bradycardia, aphasia, and orofacial dyskinesia. Behavior abnormalities became worse and there was impairment in consciousness level. Complementary investigation for rheumatologic disorders and infective diseases were negative. After the exclusion of major causes of encephalitis, NMDAr antibodies were identified in serum and CSF, and neoplasm screening did not detect any tumor. The patient was treated with cycles of IV methylprednisolone ( $30 \mathrm{mg} / \mathrm{kg} /$ day) for 5 days associated with ciclophosphamide and followed by oral prednisone ( $2 \mathrm{mg} / \mathrm{kg} / \mathrm{day}$ ). After 5 months of treatment, she has presented normal neurological examination.

\section{Discussion}

This paper characterizes the first description of patients with a serologically-proven diagnosis of anti-NMDAr encephalitis in Brazil. This entity represents an important differential diagnosis of encephalitis not only in adults, but also among the pediatric population. We observed a wide variety of symptoms among the three cases that we have described, but they share some common traits, and all of them presented normal investigation for the other common causes of encephalitis in childhood.

Based on data published in literature in the last 3 years, it is possible to estimate that anti-NMDAr encephalitis seems to be more common than any other paraneoplastic encephalitis. In the United Kingdom, for instance, a prospective study found a prevalence of $4 \%$ of anti-NMDAr encephalitis among all patients with encephalitis. ${ }^{6}$ 
Florance et al. reported the first pediatric series of anti-NMDAr encephalitis cases. ${ }^{4}$ Thirty-two patients under the age of 18 years were studied. Before this study, only sporadic cases had been reported. The first symptoms that these authors identified were behavioral changes and agitation. Seizures, speech impairment, movement disorders such as dystonia, orofacial dyskinesia, and chorea are the commonest presentations in young children. Twenty-eight patients presented with mood, behavior, or personality changes. Most patients became increasingly anxious and agitated. Only six patients had early seizures, and a total of $77 \%$ presented seizures during the evolution of symptoms. Movement disorders such as orolinguofacial dyskinesia, dystonic posture, increased tone, and choreoathetoid movements were identified in $84 \%$ of patients. Central hypoventilation was seen only in seven children. ${ }^{4}$ Even though autonomic dysfunction and hypoventilation are less frequent in childhood, those potentially fatal complications should be seriously considered as part of clinical presentation of anti-NMDAr encephalitis. Severe cardiac arrhythmia has not been described in children. 4,7 We observe in the literature of the pediatric population that anti-NMDAr encephalitis has a predominance of the female sex $(81 \%)$, the age of the onset of symptoms ranges from 23 months to 18 years (median: 14 years), and prodromal symptoms are non-specific and identified in less than $50 \%$ of the affected children. ${ }^{4}$

Therefore, the presence of movement disorders following an impairment of consciousness and/or speech disturbances in a child must direct us to the possibility of anti-NMDAr encephalitis.

The importance of acknowledging this clinical setting becomes even more relevant considering that the initial complementary tests may be normal. In adults, MRI is normal in $50 \%$ of patients and other $50 \%$ may present mild T2 or fluid-attenuated inversion-recovery (FLAIR) signal hyperintensity in hippocampus, cortex, frontobasal and insular regions, in basal ganglia and in the brainsteam. ${ }^{1}$ In children, transient FLAIR hyperintensity in one or more areas (medial temporal lobe, periventricular, cerebellar) may be present in less than one-third of cases. ${ }^{4}$ Thus, it makes the measurement of antibody titers in serum and/or CSF by ELISA essential for the diagnosis of antiNMDAr encephalitis.

Contrary to the original idea of an obligatory association between this condition and ovarian tumors, a recent review of 400 cases demonstrated that the younger the patient, the less likely a tumor will be detected. 4,7 The presence of ovarian teratomas becomes more relevant only after the age of 13 years. ${ }^{4}$ Nevertheless, screening using computed tomography scan and pelvic ultrasound are still recommended in all patients. Testicular germ-cell tumor, neuroblastoma, and Hodgkin's lymphoma are other associated tumors. If no tumor is found, the approach of this encephalitis must be only directed to immunotherapy. ${ }^{7}$
The first-line immunotherapy treatments are either IV high-dose corticosteroids, immunoglobulin, or plasma exchange. In the cases without a proper response to firstline therapy, cyclophosphamide or rituximab may be added. ${ }^{7}$ Recovery time is slow, usually taking months, but a favorable outcome, with full recovery or mild sequelae, is the rule for $75 \%$ of patients. ${ }^{2,5}$ Overall mortality rate is around $4 \%$ and is usually associated with secondary comorbidities acquired in the intensive care unit. ${ }^{7}$

Clinical signs of encephalitis, including seizures, behavior abnormalities, speech disturbances, and movement disorders are the hallmarks of anti-NMDAr encephalitis in childhood. Tumors are not usually detected by this age. The diagnosis of anti-NMDAr encephalitis must be addressed only after the exclusion of infectious and other recognizable causes of encephalitis. Emergency pediatricians, intensive care unit pediatricians and pediatric neurologists should be aware of this treatable autoimmune condition.

\section{Acknowledgements}

The authors thank Drs Denise A. De Oliveira, Pollyanna B. Lima, Karin R. Koladicz, and Larissa A. Mehl, who provided clinical assistance for the patients reported, and Dr Caio Quaio for the technical assistance. Special acknowledgement to $\mathrm{Dr}$ Josep Dalmau, who provided confirmatory tests in cases 1 and 3 .

\section{References}

1. Dalmau J, Tüzün E, Wu HY, Masjuan J, Rossi JE, Voloschin A, et al. Paraneoplastic anti-N-methyl-D-aspartate receptor encephalitis associated with ovarian teratoma. Ann Neurol. 2007;61:25-36.

2. Dalmau J, Gleichman AJ, Hughes EG, Rossi JE, Peng X, Lai M, et al. Anti-NMDA-receptor encephalitis: case series and analysis of the effects of antibodies. Lancet Neurol. 2008;7:1091-8.

3. Prüss H, Dalmau J, Harms L, Höltje M, Ahnert-Hilger G, Borowski $\mathrm{K}$, et al. Retrospective analysis of NMDA receptor antibodies in encephalitis of unknown origin. Neurology. 2010;75:1735-9.

4. Florance NR, Davis RL, Lam C, Szperka C, Zhou L, Ahmad S, et al. Anti-N-methyl-D-aspartate receptor (NMDAR) encephalitis in children and adolescents. Ann Neurol. 2009;66:11-8.

5. Wong-Kisiel LC, Ji T, Renaud DL, Kotagal S, Patterson MC, Dalmau J, et al. Response to immunotherapy in a 20-month-old boy with anti-NMDA receptor encephalitis. Neurology. 2010;74:1550-1.

6. Granerod J, Ambrose HE, Davies NW, Clewley JP, Walsh AL, Morgan $D$, et al. Causes of encephalitis and differences in their clinical presentations in England: a multicentre, population-based prospective study. Lancet Infect Dis. 2010;10:835-44.

7. Dalmau J, Lancaster E, Martinez-Hernandez E, Rosenfeld MR, Balice-Gordon R. Clinical experience and laboratory investigations in patients with anti-NMDAR encephalitis. Lancet Neurol. 2011;10:63-74.

Correspondence:

Felippe Borlot

D. Adma Jafet, 74, Cj. 121

CEP 01308-050 - São Paulo, SP - Brazil

Fax: +55 (11) 3259.8574

E-mail: felippe@dfvneuro.com.br 